\title{
Precision surgery for cancer: a new surgical concept in individual tumor biology-based image-guided surgery
}

\author{
Nayeon $\mathrm{Choi}^{1,2}$, Han-Sin Jeong ${ }^{2}$ \\ ${ }^{1}$ Department of Otorhinolaryngology-Head and Neck Surgery, The National Armed Forces Capital Hospital, Seongnam, Korea \\ ${ }^{2}$ Department of Otorhinolaryngology-Head and Neck Surgery, Samsung Medical Center, Sungkyunkwan University School of \\ Medicine, Seoul, Korea
}

Received: July 9, 2019

Revised: August 13, 2019

Accepted: August 16, 2019

Corresponding author:

Han-Sin Jeong

Department of

Otorhinolaryngology-Head

and Neck Surgery, Samsung

Medical Center, Sungkyunkwan

University School of Medicine,

81 Irwon-ro, Gangnam-gu,

Seoul 06351, Korea

Tel: +82-2-3410-3579

E-mail: hansin.jeong@gmail.com

\begin{abstract}
Surgery has long been a cornerstone of cancer treatment in many types of cancer. Traditionally, intraoperative assessment of the resection margin is largely dependent on visual inspection and palpation of tumors, with the aid of frozen section analysis. Although preoperative imaging can provide gross anatomical information, in situ translation of these images to the operation field is challenging. With the advancement of molecular imaging technology and its clinical application, the gap between preoperative radiologic images and surgical findings has been reduced through image-guided surgery. However, the imaging probes for intraoperative visualization of tumors are not individual tumor-specific. As conventional oncology has moved toward precision oncology with genomic and biological information specific to each tumor, image-guided surgery should also shift toward tumor biology-based image-guided surgery, so-called precision surgery for cancer. In precision cancer surgery, tumors should be analyzed molecularly and genetically to select the optimal imaging probes for individual tumors before surgical resection, beyond the use of predetermined imaging probes for certain types of cancer. This will raise the likelihood of meeting the surgical goals of cancer treatment. In summary, precision cancer surgery can be defined as individual tumor biology-based image-guided surgery.
\end{abstract}

Keywords: Molecular imaging; Neoplasms; Precision oncology; Surgery; Surgery, computer-assisted
This is an Open Access article distributed under the terms of the Creative Commons Attribution Non-Commercial License (http:// creativecommons.org/licenses/ by-nc/4.0/).

\section{INTRODUCTION}

Surgery has long been a cornerstone of cancer treatment in many types of cancer. The main goal of surgical cancer treatment is to completely remove the tumor without remnant cancer cells. Achievement of tumor-free margins is essential to reduce the risk of local recurrence; meanwhile positive resection margins (the presence of cancer cells at the resection margin) is one of the most significant predictors for recurrence and shortened survival in various cancers [1-4]. Furthermore, poor treatment outcomes related to positive resection margins may not 
improve after salvage surgery or adjuvant treatment [1-5].

In this manuscript, we reviewed the use of optical tools during surgery with regard to the goals of surgical resection for cancer treatment and sought to define a new surgical concept: precision cancer surgery as an extension of image-guided surgery.

\section{LIMITATIONS OF CURRENT SURGICAL TREATMENTS}

Traditionally, intraoperative assessment of the resection margin is largely dependent on visual inspection and palpation of tumors, with the aid of frozen section analysis. Although preoperative imaging such as computed tomography, ultrasonography (US), and magnetic resonance imaging (MRI) can provide gross anatomical information, in situ translation of these images to the operation field is challenging. Even with intraoperative frozen section analysis, overall diagnostic accuracy is not high due to sampling error and limited amount of samples and examination [6,7]. Due to these factors, the incidence of close (a less than 5-mm safety margin around tumors) and positive margins is as high as $40 \%$ in head and neck cancers [8].

\section{IMAGE GUIDANCE FOR PRECISE CANCER SURGERY}

With the advancement of molecular imaging technology and its clinical application, the gap between preoperative radiologic images and surgical findings has been reduced through image-guided surgery [9-11]. The primary purpose of image-guided surgery is to visually differentiate tumor cells from the surrounding tissues in real time, which enables complete resection and preservation of normal function to the greatest extent possible [12].

\section{Conventional imaging-guided surgery}

Intraoperative delineation of tumors using conventional anatomical imaging modalities has been applied to tumor ablative surgery for several solid cancers. Glioblastoma is a highly infiltrative brain tumor with poorly defined tumor boundaries. Preoperative MRI is limited to defining tumor margin clearly during surgery because of the brain shift and swelling that occurs during surgery [13]. To overcome these shortcomings of preoperative imaging, intraoperative MRI has been introduced into the neurosurgical field. Many studies have reported that intraoperative MRI-guided surgery improves resection quality without increasing neurological complications and has a positive impact on survival [13-15]. In a randomized controlled trial, intraoperative MRI offered $96 \%$ total resection in comparison with $68 \%$ without it [13]. However, the need for multiple resources for intraoperative MRI may be a stumbling block to clinical application in other types of cancers.

Breast conservation surgery has been recognized as a standard surgery for early breast cancer. Similar to other solid cancers, positive resection margins are a significant poor prognostic factor for recurrence and survival [16-18]. A randomized clinical trial comparing intraoperative US-guided surgery to palpation-guided surgery revealed significant improvement in securing negative resection margins with US during surgery ( $3 \%$ vs. $17 \%$ positive resection margins favoring US-guided surgery) [18]. In addition, US-guided surgery reduced the resection volume with good margin status, suggesting that it enabled more accurate surgery.

In short, intraoperative radiology or US-guided surgery has the potential to improve the primary goal of surgery using anatomical information during surgery. However, it has some limitations such as the low anatomical resolution of conventional imaging for surgery, inability to capture anatomical displacement or distortion during surgical manipulation, difficulty of real-time imaging during surgery (except intraoperative US), and a lack of cancer-specific information.

\section{Fluorescence-guided surgery}

Fluorescence optical imaging with various imaging probes and molecular targeting materials has been introduced for real-time image-guided surgery to overcome the drawbacks of conventional imaging. These technological developments enable the surgeon to remove the cancer at a submillimeter level $[12,19]$. The characteristics of cancer cells including increased transformed optical properties, growth factors, angiogenesis, and proteolysis have been utilized in fluorescence optical image-guided surgery [20]. This allows real-time feedback during surgery even in the surgical wound bed. As a result, it can provide wide surgical field images, and can detect microscopic residual disease.

\section{Conventional fluorescence-guided surgery}

In a fluorescence-guided surgery, tumor-specific signals are the main target for visualization to discriminate between tumor and normal tissues [21]. Thus, signal to background ratio (or tumor to background ratio) is a key component of optical imaging. Molecules with fluorescent light emission are known as fluorophores, whereas molecules with light ab- 
sorption are called chromophores [20]. The representative chromophore in cancer imaging is hemoglobin, which absorbs light spectra less than $600 \mathrm{~nm}$ in wavelength. Water and lipids absorb light spectra over $900 \mathrm{~nm}$ [20]. Fluorescent imaging using the visible light spectrum (400- to 600-nm wavelength) has high nonspecific background light with scattering and a low tumor to background ratio [22], which frequently fails to meet the imaging criteria (tumor to background ratio > 3) [23].

\section{Near-infrared fluorescence imaging}

A light spectrum between 650 and $900 \mathrm{~nm}$ is more desirable for fluorescence imaging, because less absorption in normal tissue and a relatively high tumor to background ratio [24]. In addition, this near-infrared light spectrum has high tissue penetration ( 5 to $10 \mathrm{~mm}$ ) with little interference from intrinsic fluorescence [10]. Thus, nonspecific fluorescence can be minimized and tumors can be delineated more clearly during surgery. In line with the improvement in near-infrared fluorescent imaging, several imaging systems have been introduced in the clinical setting to visualize near-infrared signals [25-27].

\section{FLUORESCENT PROBE FOR IMAGE- GUIDED SURGERY}

Indocyanine green (ICG) is the most popular imaging agent for image-guided surgery. IGC emits a light spectrum of 700 to $800 \mathrm{~nm}$ (a near-infrared fluorescent contrast agent), and has been approved by the U.S. Food and Drug Administration for surgery [11]. It is cleared by the liver and has been used for a long time to evaluate the clearance function of the liver and to image blood vessels (angiography). After binding with plasma proteins, ICG is retained in tumor tissues through the increased permeability of tumor vessels and lymphatics [28]. In a trial of ICG, it was able to identify small metastatic tumors of hepatocellular carcinoma [28]. The fluorescent margin of the tumor was demarcated from surrounding normal tissues during several hours to days after intravenous injection of ICG [28]. Many studies reported the clinical application of ICG for various solid tumors including colorectal, gastric, and head and neck cancer $[10,20,21,29]$. In addition to ICG, many fluorescent dyes including Cy5.5, Cy7, IRdye800 $\mathrm{CW}$, and quantum dots have been used for near-infrared imaging and image-guided surgery $[12,20]$.

\section{BIOLOGIC MARKERS FOR IMAGE-GUIDED SURGERY}

Even with a high tumor to background ratio, the above fluorescent imaging probes are not tumor-specific, accumulating in tumor tissues in a passive manner. Thus, several biological signals have been incorporated into molecular tumor imaging to enhance tumor-specific uptake intraoperatively. Folate receptor-alpha [30], epidermal growth factor receptor [31], HER2/neu [32], prostate-specific membrane antigen [33], transferrin receptor [34], and carcinoembryonic antigen 19-9 [35] have been studied to distinguish tumor from normal tissue in various types of cancer.

For example, in breast cancer, ICG with trastuzumab (monoclonal antibody against HER2 receptor) was investigated to visualize margins and to classify molecular subtype during surgery [36]. Adhesion molecule alpha-v-beta-3 integrin can also be an imaging target for tumor delineation by Cy5.5 or IRDye800 CW conjugate with an antibody against alpha-v-beta-3 integrin $[26,37,38]$.

A randomized controlled trial of 5-aminolevulinic acid (5ALA) was a landmark study of an imaging probe that improved progression-free survival in glioblastoma patients [39]. However, this study did not provide preoperative molecular or genetic information regarding brain tumors with regard to whether cancer tissue had 5-ALA susceptibility.

The tumor environment is aberrantly transformed by cancer cells. This causes dysregulated extra- and intracellular $\mathrm{pH}$ [40], hypoxia [41], increased secretion of matrix metalloproteinases [42], cathepsins [43], and gamma-glutamyl transpeptidase [44], which are potential targets for tumor-specific image-guided surgery.

In head and neck cancers, near-infrared imaging dye of IRDye800 CW conjugated with anti-epidermal growth factor receptor (panitumumab) has been used in head and neck squamous cell carcinoma [45]. In a phase I trial, cetuximab (anti-epidermal growth factor receptor [EGFR] antibody)IRDye800 was shown to improve surgical resection more precisely with a high tumor to background ratio [46]. Tumor was sharply demarcated from surrounding normal tissues within $1 \mathrm{~mm}$, and fluorescence was strongly correlated with the location of the tumor, as confirmed by biopsy [47]. Another study of cetuximab-IRDye800 for head and neck cancer also showed the safety and tumor specificity of image-guided surgery [46]. This study enrolled patients with biopsy-proven head and neck squamous cell carcinoma, not specified into positive epidermal growth factor tumor. 
Transferrin receptor is a poor prognostic indicator and is overexpressed in head and neck cancer. An in vivo study showed that the maximum fluorescence signal of the tumors occurred between 90 and 120 minutes after injection and a high tumor to background ratio was achieved [34]. Another study using Cy5.5 conjugated anti-vascular endothelial growth factor antibody (bevacizumab) in an animal experiment also demonstrated the feasibility of image-guided surgery for head and neck cancers using this probe [48].

\section{NEW DEFINITION OF PRECISION CANCER SURGERY}

Tumors may have different phenotypes and characteristics according to anatomical location, histologic subtype, previous treatment, tumor burden (stage) and chronological effect [49]. This can be a major hurdle to choosing the best-fit imaging probe that correctly or accurately visualizes target tumor cells in precision image-guided surgery. Thus, it should be further improved with a tailored method reflecting individual tumor-specific biological changes. In this article, we suggest a new surgical concept, individual tumor biology-based (individualized) image-guided surgery, and investigate its potential significance in surgical oncology.

Traditionally, cancer treatment has been based on tumor location (primary site) and histopathology [50]. However, recent development of genomic and molecular technologies revealed that cancers from the same origin and histopathol-

Conventional oncology for recurrent/metastatic cancer

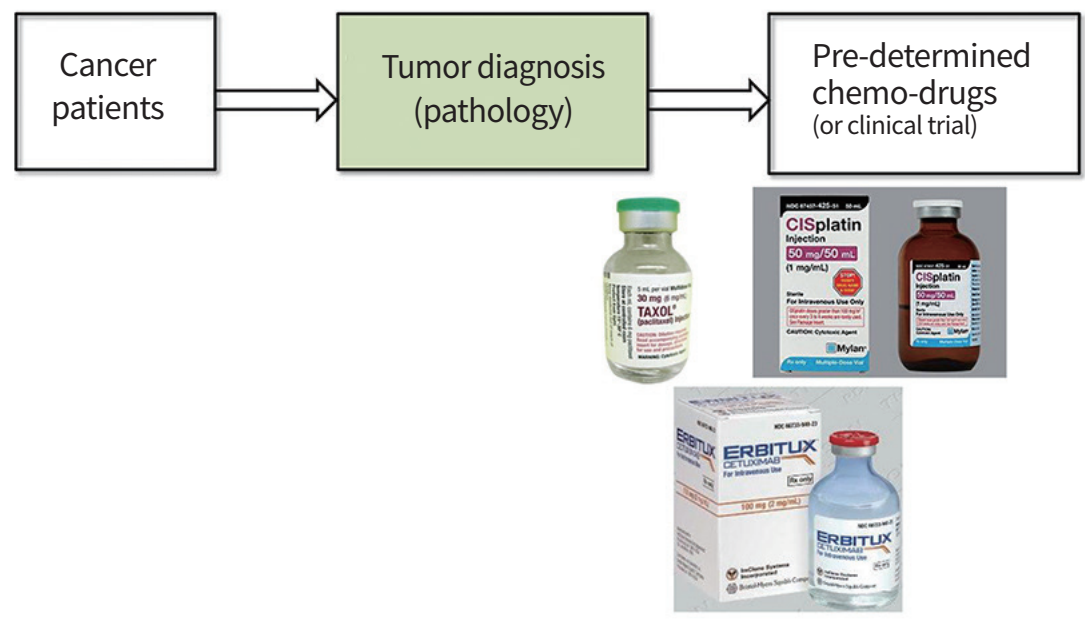

Precision oncology for recurrent/metastatic cancer

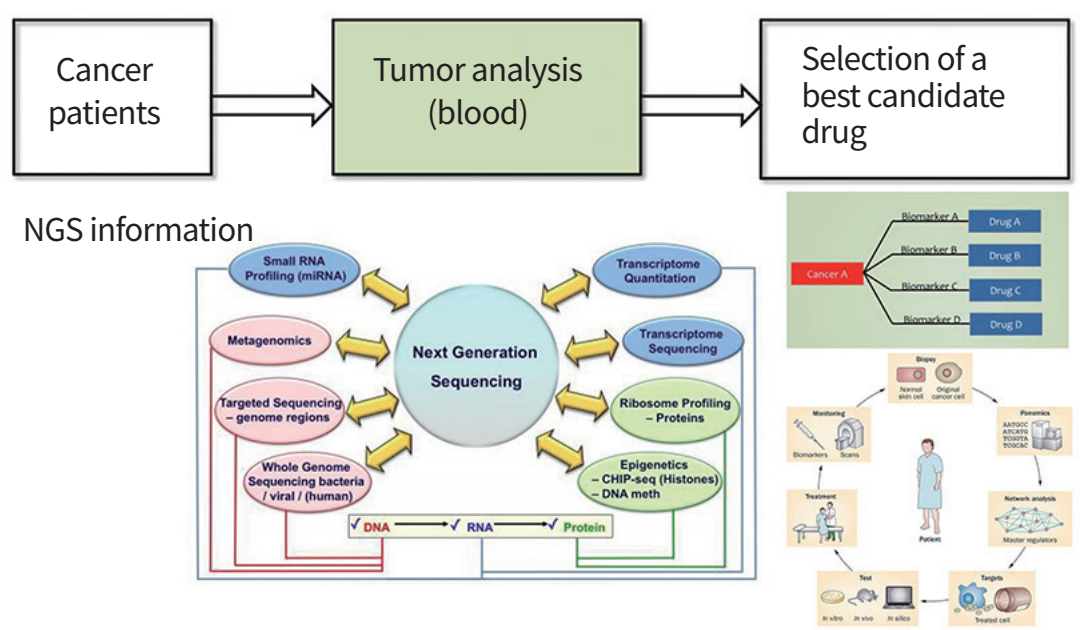

Fig. 1. Basic concept of precision oncology (precision medicine) compared with the conventional oncology. NGS, next generation sequencing. 
ogy can have molecular diversity, which may result in treatment resistance or failure [51]. With cancer genomic atlas, many clinicians and researchers expect that a rational selection of target agents for specific genetic and molecular alterations of cancer, even from the diverse origin sites [51,52].

This would accelerate the feasibility of prevention, early detection, and patient-tailored treatment of various types of cancers. Translation of molecular and genomic data into cancer treatments enables personalized cancer medicine with specific target agents [53].

Precision medicine in a basket clinical trial linked molecular targets specific to the genetic aberrations of cancer and target drugs, irrespective of the tumor origin [54]. Several genome-based cancer medicines have been used in clinical settings, including trastuzumab for HER2 mutation in breast cancer [55], imatinib for tyrosine kinase KIT (CD117) aberration in gastrointestinal stromal tumors [56], and erlotinib for EGFR in lung cancer [57]. However, precision oncology does not promise clinical benefits; a previous report of 2,000 consecutive pa- tients showed that only $6.4 \%$ of patients had actionable alterations fitting genotype-matched trials [58]. Nonetheless, rapid accumulation of genetic information, technology, and drugs has improved the clinical outcomes of several target agents.

The main purpose of cancer surgery is to identify the extent of tumor accurately and remove tumors completely with minimal loss of physiological function. In conventional oncological surgery, surgeons have relied on visual inspection with white light and tactile sensations during surgery, as mentioned before. Clinical application of intraoperative molecular imaging has improved the outcomes of surgical cancer treatments [59]. However, the imaging probes for intraoperative visualization of tumors are not individual tumor-specific. As conventional oncology has moved toward precision oncology with genomic and biological information specific to each tumor, image-guided surgery should also shift toward tumor biology-based image-guided surgery, socalled precision surgery for cancer (Figs. 1, 2). This is a simple concept with regard to more accurate image-guided surgery.

Conventional surgery for resectable cancer

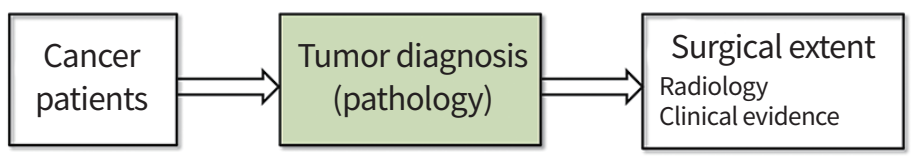

No tumor specific or patient specific surgery

Cf.

Image-guided surgery based on predetermined biomarker issues: It does not reflect individualized tumor characteristics, patient specificity and tumor heterogeneity.

Precision surgery for resectable cancer

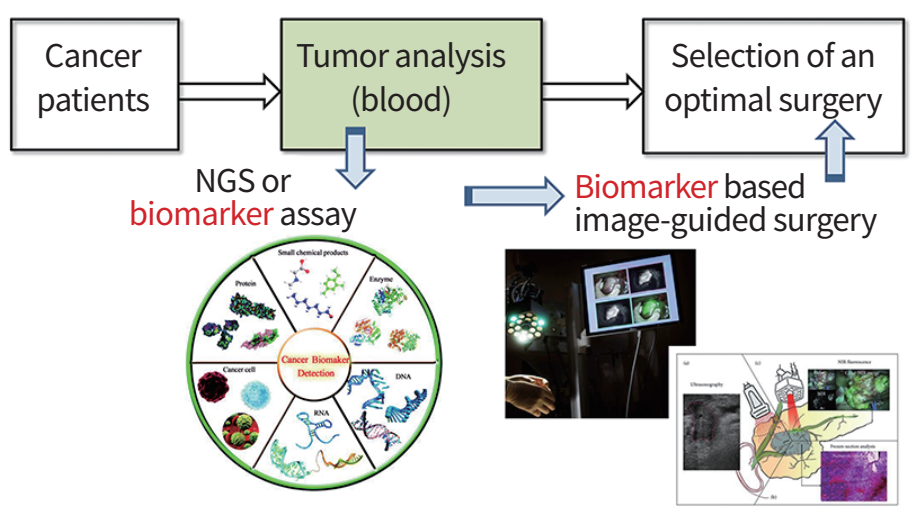

Fig. 2. Translation of a precision oncology concept to the precision cancer surgery. In precision cancer surgery, tumors are analyzed molecularly and genetically to select the optimal imaging probes for individual tumors before surgical resection, beyond the use of predetermined imaging probes for certain types of cancer. NGS, next generation sequencing. 
In precision cancer surgery, tumors should be analyzed molecularly and genetically to select the optimal imaging probes for individual tumors before surgical resection, beyond the use of predetermined imaging probes for certain types of cancer. This will raise the likelihood of meeting the surgical goals of cancer treatment.

\section{CONCLUSION}

In this manuscript, we reviewed the current status of optical tools during surgery for cancer treatment and tried to define a new concept of precision cancer surgery. Based on the previous and ongoing works of image-guided surgery, precision cancer surgery can be defined as individual tumor biology-based image-guided surgery. Surgical treatment for cancer will be advancing to a more accurate and precise surgery through understanding and application of individual tumor biology near future.

\section{CONFLICTS OF INTEREST}

No potential conflict of interest relevant to this article was reported.

\section{ACKNOWLEDGMENTS}

This research was supported by a grant from the National Research Foundation (NRF) funded by the Korean government (2018R1A2B6002920).

\section{ORCID}

Nayeon Choi https://orcid.org/0000-0002-0962-0594

Han-Sin Jeong https://orcid.org/0000-0003-4652-0573

\section{REFERENCES}

1. Hinni ML, Ferlito A, Brandwein-Gensler MS, Takes RP, Silver CE, Westra WH, et al. Surgical margins in head and neck cancer: a contemporary review. Head Neck 2013;35: 1362-70.

2. Singletary SE. Surgical margins in patients with early-stage breast cancer treated with breast conservation therapy. Am J Surg 2002;184:383-93.

3. Meric F, Mirza NQ, Vlastos G, Buchholz TA, Kuerer HM, Babiera GV, et al. Positive surgical margins and ipsilateral breast tumor recurrence predict disease-specific survival after breast-conserving therapy. Cancer 2003;97:926-33.

4. Dotan ZA, Kavanagh K, Yossepowitch O, Kaag M, Olgac S, Donat $\mathrm{M}$, et al. Positive surgical margins in soft tissue following radical cystectomy for bladder cancer and cancer specific survival. J Urol 2007;178:2308-12.

5. Haque R, Contreras R, McNicoll MP, Eckberg EC, Petitti DB. Surgical margins and survival after head and neck cancer surgery. BMC Ear Nose Throat Disord 2006;6:2.

6. DiNardo LJ, Lin J, Karageorge LS, Powers CN. Accuracy, utility, and cost of frozen section margins in head and neck cancer surgery. Laryngoscope 2000;110:1773-6.

7. Black C, Marotti J, Zarovnaya E, Paydarfar J. Critical evaluation of frozen section margins in head and neck cancer resections. Cancer 2006;107:2792-800.

8. McMahon J, O'Brien CJ, Pathak I, Hamill R, McNeil E, Hammersley N, et al. Influence of condition of surgical margins on local recurrence and disease-specific survival in oral and oropharyngeal cancer. $\mathrm{Br} \mathrm{J}$ Oral Maxillofac Surg 2003;41:224-31.

9. Labadie RF, Davis BM, Fitzpatrick JM. Image-guided surgery: what is the accuracy? Curr Opin Otolaryngol Head Neck Surg 2005;13:27-31.

10. Gioux S, Choi HS, Frangioni JV. Image-guided surgery using invisible near-infrared light: fundamentals of clinical translation. Mol Imaging 2010;9:237-55.

11. Keereweer S, Kerrebijn JD, van Driel PB, Xie B, Kaijzel EL, Snoeks TJ, et al. Optical image-guided surgery: where do we stand? Mol Imaging Biol 2011;13:199-207.

12. Iqbal $\mathrm{H}$, Pan $\mathrm{Q}$. Image guided surgery in the management of head and neck cancer. Oral Oncol 2016;57:32-9.

13. Senft C, Bink A, Franz K, Vatter H, Gasser T, Seifert V. Intraoperative MRI guidance and extent of resection in glioma surgery: a randomised, controlled trial. Lancet Oncol 2011; 12:997-1003.

14. Senft C, Schoenes B, Gasser T, Platz J, Bink A, Franz K, et al. Feasibility of intraoperative MRI guidance for craniotomy and tumor resection in the semisitting position. $J$ Neurosurg Anesthesiol 2011;23:241-6.

15. Claus EB, Horlacher A, Hsu L, Schwartz RB, Dello-lacono $D$, Talos F, et al. Survival rates in patients with low-grade glioma after intraoperative magnetic resonance image guidance. Cancer 2005;103:1227-33.

16. Yu CC, Chiang KC, Kuo WL, Shen SC, Lo YF, Chen SC. Low re-excision rate for positive margins in patients treated with ultrasound-guided breast-conserving surgery. Breast 2013;22:698-702.

17. Dunne C, Burke JP, Morrow M, Kell MR. Effect of margin 
status on local recurrence after breast conservation and radiation therapy for ductal carcinoma in situ. J Clin Oncol 2009;27:1615-20.

18. Krekel NM, Haloua MH, Lopes Cardozo AM, de Wit RH, Bosch AM, de Widt-Levert LM, et al. Intraoperative ultrasound guidance for palpable breast cancer excision (COBALT trial): a multicentre, randomised controlled trial. Lancet Oncol 2013;14:48-54.

19. Savariar EN, Felsen CN, Nashi N, Jiang T, Ellies LG, Steinbach $\mathrm{P}$, et al. Real-time in vivo molecular detection of primary tumors and metastases with ratiometric activatable cell-penetrating peptides. Cancer Res 2013;73:85564.

20. Rosenthal EL, Warram JM, Bland KI, Zinn KR. The status of contemporary image-guided modalities in oncologic surgery. Ann Surg 2015;261:46-55.

21. Wang C, Wang Z, Zhao T, Li Y, Huang G, Sumer BD, et al. Optical molecular imaging for tumor detection and image-guided surgery. Biomaterials 2018;157:62-75.

22. DaCosta RS, Wilson BC, Marcon NE. Fluorescence and spectral imaging. ScientificWorldJournal 2007;7:2046-71.

23. Keereweer S, Sterenborg HJ, Kerrebijn JD, Van Driel PB, Baatenburg de Jong RJ, Lowik CW. Image-guided surgery in head and neck cancer: current practice and future directions of optical imaging. Head Neck 2012;34:120-6.

24. Chance B. Near-infrared images using continuous, phase-modulated, and pulsed light with quantitation of blood and blood oxygenation. Ann N Y Acad Sci 1998; 838:29-45.

25. Day KE, Beck LN, Deep NL, Kovar J, Zinn KR, Rosenthal EL. Fluorescently labeled therapeutic antibodies for detection of microscopic melanoma. Laryngoscope 2013; 123:2681-9.

26. Chi C, Du Y, Ye J, Kou D, Qiu J, Wang J, et al. Intraoperative imaging-guided cancer surgery: from current fluorescence molecular imaging methods to future multi-modality imaging technology. Theranostics 2014;4:1072-84.

27. Sugie T, Sawada T, Tagaya N, Kinoshita T, Yamagami K, Suwa $\mathrm{H}$, et al. Comparison of the indocyanine green fluorescence and blue dye methods in detection of sentinel lymph nodes in early-stage breast cancer. Ann Surg Oncol 2013;20:2213-8.

28. Ishizawa T, Fukushima N, Shibahara J, Masuda K, Tamura S, Aoki T, et al. Real-time identification of liver cancers by using indocyanine green fluorescent imaging. Cancer 2009; 115:2491-504.

29. Schaafsma BE, Mieog JS, Hutteman M, van der Vorst JR,
Kuppen PJ, Lowik CW, et al. The clinical use of indocyanine green as a near-infrared fluorescent contrast agent for image-guided oncologic surgery. J Surg Oncol 2011; 104:323-32.

30. van Dam GM, Themelis G, Crane LM, Harlaar NJ, Pleijhuis RG, Kelder W, et al. Intraoperative tumor-specific fluorescence imaging in ovarian cancer by folate receptor- $\alpha$ targeting: first in-human results. Nat Med 2011;17:1315-9.

31. Ke S, Wen X, Gurfinkel M, Charnsangavej C, Wallace S, Sevick-Muraca EM, et al. Near-infrared optical imaging of epidermal growth factor receptor in breast cancer xenografts. Cancer Res 2003;63:7870-5.

32. Koyama Y, Hama Y, Urano Y, Nguyen DM, Choyke PL, Kobayashi $\mathrm{H}$. Spectral fluorescence molecular imaging of lung metastases targeting HER2/neu. Clin Cancer Res 2007;13:2936-45.

33. Nakajima T, Mitsunaga M, Bander NH, Heston WD, Choyke PL, Kobayashi H. Targeted, activatable, in vivo fluorescence imaging of prostate-specific membrane antigen (PSMA) positive tumors using the quenched humanized J591 antibody-indocyanine green (ICG) conjugate. Bioconjug Chem 2011;22:1700-5.

34. Shan L, Hao Y, Wang S, Korotcov A, Zhang R, Wang T, et al. Visualizing head and neck tumors in vivo using near-infrared fluorescent transferrin conjugate. Mol Imaging 2008;7:42-9.

35. McElroy M, Kaushal S, Luiken GA, Talamini MA, Moossa AR, Hoffman RM, et al. Imaging of primary and metastatic pancreatic cancer using a fluorophore-conjugated anti-CA19-9 antibody for surgical navigation. World J Surg 2008;32:1057-66.

36. Sano K, Mitsunaga M, Nakajima T, Choyke PL, Kobayashi $\mathrm{H}$. In vivo breast cancer characterization imaging using two monoclonal antibodies activatably labeled with near infrared fluorophores. Breast Cancer Res 2012;14:R61.

37. Ye $\mathrm{Y}$, Chen $\mathrm{X}$. Integrin targeting for tumor optical imaging. Theranostics 2011;1:102-26.

38. Wenk CH, Ponce F, Guillermet S, Tenaud C, Boturyn D, Dumy $P$, et al. Near-infrared optical guided surgery of highly infiltrative fibrosarcomas in cats using an anti-avß3 integrin molecular probe. Cancer Lett 2013;334:188-95.

39. Stummer W, Pichlmeier U, Meinel T, Wiestler OD, Zanella F, Reulen HJ, et al. Fluorescence-guided surgery with 5-aminolevulinic acid for resection of malignant glioma: a randomised controlled multicentre phase III trial. Lancet Oncol 2006;7:392-401.

40. Zhao T, Huang G, Li Y, Yang S, Ramezani S, Lin Z, et al. A 
transistor-like $\mathrm{pH}$ nanoprobe for tumour detection and image-guided surgery. Nat Biomed Eng 2016;1:0006.

41. Petrova V, Annicchiarico-Petruzzelli M, Melino G, Amelio I. The hypoxic tumour microenvironment. Oncogenesis 2018:7;10.

42. Cho HJ, Lee S, Park SJ, Lee YD, Jeong K, Park JH, et al. Tumor microenvironment-responsive fluorogenic nanoprobe for ratiometric dual-channel imaging of lymph node metastasis. Colloids Surf B Biointerfaces 2019;179: 9-16.

43. Weissleder R, Tung CH, Mahmood U, Bogdanov A Jr. In vivo imaging of tumors with protease-activated near-infrared fluorescent probes. Nat Biotechnol 1999;17:375-8.

44. Urano Y, Sakabe M, Kosaka N, Ogawa M, Mitsunaga M, Asanuma D, et al. Rapid cancer detection by topically spraying a $\gamma$-glutamyltranspeptidase-activated fluorescent probe. Sci Transl Med 2011;3:110ra119.

45. Heath CH, Deep NL, Sweeny L, Zinn KR, Rosenthal EL. Use of panitumumab-IRDye 800 to image microscopic head and neck cancer in an orthotopic surgical model. Ann Surg Oncol 2012;19:3879-87.

46. Rosenthal EL, Warram JM, de Boer E, Chung TK, Korb ML, Brandwein-Gensler M, et al. Safety and tumor specificity of cetuximab-IRDye800 for surgical navigation in head and neck cancer. Clin Cancer Res 2015;21:3658-66.

47. de Boer E, Warram JM, Tucker MD, Hartman YE, Moore LS, de Jong JS, et al. In vivo fluorescence immunohistochemistry: localization of fluorescently labeled cetuximab in squamous cell carcinomas. Sci Rep 2015;5:10169.

48. Withrow KP, Newman JR, Skipper JB, Gleysteen JP, Magnuson JS, Zinn K, et al. Assessment of bevacizumab conjugated to Cy5.5 for detection of head and neck cancer xenografts. Technol Cancer Res Treat 2008;7:61-6.

49. Ludwig JA, Weinstein JN. Biomarkers in cancer staging, prognosis and treatment selection. Nat Rev Cancer 2005; 5:845-56.

50. National Comprehensive Cancer Network. NCCN Clinical
Practice Guidelines in Oncology (NCCN Guidelines ${ }^{\circledR}$ ) Head and Neck Cancers Version 2.2017 [Internet]. Plymouth Meeting (PA): National Comprehensive Cancer Network; c2017 [cited 2019 August 28]. Available from: www. NCCN.org.

51. Cancer Genome Atlas Research Network, Weinstein JN, Collisson EA, Mills GB, Shaw KR, Ozenberger BA, et al. The Cancer Genome Atlas Pan-Cancer analysis project. Nat Genet 2013;45:1113-20.

52. Biankin AV, Piantadosi S, Hollingsworth SJ. Patient-centric trials for therapeutic development in precision oncology. Nature 2015;526:361-70.

53. Grimson WL, Ettinger GJ, White SJ, Lozano-Perez T, Wells WM, Kikinis R. An automatic registration method for frameless stereotaxy, image guided surgery, and enhanced reality visualization. IEEE Trans Med Imaging 1996;15:12940.

54. West HJ. Novel precision medicine trial designs: umbrellas and baskets. JAMA Oncol 2017;3:423.

55. Piccart-Gebhart MJ, Procter M, Leyland-Jones B, Goldhirsch A, Untch M, Smith I, et al. Trastuzumab after adjuvant chemotherapy in HER2-positive breast cancer. $\mathrm{N}$ Engl J Med 2005;353:1659-72.

56. Heinrich MC, Corless CL, Demetri GD, Blanke CD, von Mehren $\mathrm{M}$, Joensuu $\mathrm{H}$, et al. Kinase mutations and imatinib response in patients with metastatic gastrointestinal stromal tumor. J Clin Oncol 2003;21:4342-9.

57. Tsao MS, Sakurada A, Cutz JC, Zhu CQ, Kamel-Reid S, Squire J, et al. Erlotinib in lung cancer: molecular and clinical predictors of outcome. N Engl J Med 2005;353: 133-44.

58. Meric-Bernstam F, Brusco L, Shaw K, Horombe C, Kopetz $\mathrm{S}$, Davies MA, et al. Feasibility of large-scale genomic testing to facilitate enrollment onto genomically matched clinical trials. J Clin Oncol 2015;33:2753-62.

59. Hussain T, Nguyen QT. Molecular imaging for cancer diagnosis and surgery. Adv Drug Deliv Rev 2014;66:90-100. 